Adewuya, A. O., Ola, B. A., Aloba, O. O., et al (2006b) Anxiety disorders among Nigerian women in late pregnancy: a controlled study. Archives of Women's Mental Health, 9, 325-328.

Adewuya, A. O., Ologun, Y. A. \& Ibigbami, O. S. (2006c) Post-traumatic stress disorder after childbirth in Nigerian women: prevalence and risk factors. British Journal of Obstetrics and Gynaecology, 113, 284-288.

Adewuya, A. O., Ola, B. A., Dada, A. O., et al (2006d) Validation of the Postnatal Depression Scale as a screening tool for depression in late pregnancy among Nigerian women. Journal of Psychosomatic Obstetrics and Gynecology, 27, 267-272.

Adewuya, A. O., Ola, B. A., Aloba, O. O., et al (2008) Impact of postnatal depression on infants' growth in Nigeria. Journal of Affective Disorders, 108, 191-193.

Loto, O. M., Adewuya, A. O., Ajenifuja, O. K., et al (2009) The effect of caesarean section on self-esteem amongst primiparous women in south-western Nigeria: a case control study. Journal of Maternal-Fetal and Neonatal Medicine, 1-5 (epub ahead of print).

\title{
The Stree Arogya Shodh: investigating gynaecological morbidities and women's mental health in India
}

\author{
Vikram Patel
}

Professor of International Mental Health and Wellcome Trust Senior Clinical Research Fellow in Tropical Medicine, London School of Hygiene and Tropical Medicine and Sangath, Goa, India, email vikram.patel@lshtm.ac.uk

\begin{abstract}
G ynaecological complaints have long been associated with poor mental health, in particular depression and anxiety, in higher-income countries. Lower abdominal pain (both menstrual and non-menstrual) and pelvic pain are considered the hallmark gynaecological symptoms associated with somatoform, stress-related and neurotic disorders (the 'common mental disorders', CMDs). Cultural and social factors heavily influence the expression of somatic symptoms and metaphors for emotional distress, but little is known about gynaecological psychosomatic clinical syndromes in non-Western cultures, despite the enormous global health significance of gynaecological symptoms. This paper considers the specific example of the syndrome of abnormal vaginal discharge (AVD) in India.
\end{abstract}

\section{Abnormal vaginal discharge}

Community studies in India show that about half of all women have at least one current gynaecological complaint, of which AVD is the most common. It is characterised by the complaint of vaginal discharge being 'abnormal' on account of increased volume, colour (most often 'white') or malodour. The complaint is associated with considerable self-reported disability, health seeking and associated costs of illness. The standard clinical approach to AVD is syndromic, which is based on the assumption that the complaint is the result of sexual tract infections (STIs), notably Chlamydia trachomatis infection, gonorrhoea or trichomoniasis. Sexual tract infections are potential markers for unsafe sexual behaviour and increased vulnerability to HIV/AIDS, and are associated with adverse health outcomes such as infertility, intrauterine growth retardation, pelvic inflammatory disease, stillbirth and premature labour. Although identification and treatment of STIs are priorities in reproductive health programmes, their diagnosis is hampered by the absence of cheap, simple and accurate diagnostic tests. Thus, the World Health Organization developed the syndromic management guidelines for the treatment of STIs in resource-poor settings. The primary target for the syndromic management of STIs is the complaint of AVD. However, the evidence suggests that the association between the complaint of AVD and the presence of STIs is weak (Patel \& Oomman, 1999). A review of community surveys from South Asia reported that although over half of women have gynaecological complaints, less than half of them have laboratory-confirmed STIs. Studies in clinical settings in South Asia, where one might expect a higher proportion of women with STIs, also show that only about a quarter of women with AVD have a laboratory-confirmed STI. Thus, the current syndromic approach, which assumes AVD to be a marker for STI, is ineffective for the management of both the clinical syndrome and STI. The economic cost of syndromic management per true case of STI is enormous, while the social cost of incorrectly labelling a woman as having an STI is incalculable (Patel \& Oomman, 1999).

\section{The Stree Arogya Shodh}

Why so many women in South Asia complain of AVD was the primary research question of the Stree Arogya Shodh (SAS; 'women's health study' in the Konkani language). This study, funded by the Wellcome Trust, was carried out in the state of Goa on the west coast of India between 2001 and 2004. The SAS was implemented through a partnership between a UK academic institution (the London School of Hygiene and Tropical Medicine), the government of Goa's health services department and Sangath, a Goan community-based nongovernmental organisation pioneering mental health research projects (see http://www.sangath.com). The study was one of the largest population-based cohort studies of women's health in South Asia; it involved the collection of both interview-based 
data and biological specimens. Altogether, 3000 women aged 18-45 years were randomly selected from the family registers of a primary health centre. Participants who consented were contacted by the research team on three occasions over a 1-year period: at enrolment, and 6 and 12 months later. Participation entailed a face-to-face interview (which lasted $40 \mathrm{~min}$ to $1 \mathrm{~h}$ ) and collection of biological specimens (urine sample and/or vaginal swabs) for the diagnosis of STIs. The interview included the Revised Clinical Interview Schedule, a structured interview for use by lay interviewers which generates ICD-10 diagnoses of depression and anxiety disorders, and a checklist for somatic symptoms considered key features of somatoform disorders. In total, 2494 of the 3000 randomly selected women (i.e. over $80 \%$ ) consented to participate, and over $80 \%$ of these women completed the 6 - and 12 -month reviews.

The aim of the study was to test the hypothesis that CMDs are associated with AVD. The findings of the study have been published in a number of key publications, which form the basis of this commentary. These publications report the baseline prevalence of AVD and CMDs and their association with each other (Patel et al, 2006a), as well as the risk factors for new episodes of AVD and CMDs observed during the follow-up phase (Patel et al, 2006b,c). Nested in the cohort was a qualitative study investigating the illness narratives of women with AVD (Patel et al, 2008).

\section{Key findings}

The baseline prevalence rates and 12-month incidence rates of the three health conditions are reported in Table 1. Abnormal vaginal discharge was confirmed to be a common complaint. In both cross-sectional and longitudinal analyses, independent associations were found (after adjustment for confounders) between AVD at baseline and poor mental health (both CMDs and other somatic complaints). In both cross-sectional and longitudinal analyses, younger age and indicators of social and gender disadvantage were associated with AVD. In turn, AVD at baseline was strongly associated with the onset of new episodes of CMDs. Sexually transmitted infections were not associated with AVD at baseline or longitudinally (though bacterial vaginosis, a little-understood endogenous reproductive tract infection, was).

In the qualitative study, women associated the onset of the complaint of AVD most often with menarche and the onset of menses. The complaint was felt to be closely associated with other physical complaints, especially weakness,

Table 1 Baseline prevalence and 12-month onset rates (with $95 \%$ confidence intervals) of health conditions in the women participating in the Stree Arogya Shodh

\begin{tabular}{|lll|}
\hline Health problem & Baseline prevalence & $\begin{array}{l}12 \text {-month onset } \\
\text { rates }\end{array}$ \\
\hline $\begin{array}{c}\text { Complaint of } \\
\text { abnormal vaginal } \\
\text { discharge }\end{array}$ & $14.5 \%(13.1-15.9 \%)$ & $6.9 \%(5.9-8.0 \%)$ \\
$\begin{array}{c}\text { Sexually transmitted } \\
\text { infection }\end{array}$ & $4.2 \%(3.4-5.0 \%)$ & $2.9 \%(2.3-3.7 \%)$ \\
$\begin{array}{c}\text { Common mental } \\
\text { disorder }\end{array}$ & $6.6 \%(5.7-7.6 \%)$ & $1.8 \%(1.3-2.4 \%)$ \\
\hline
\end{tabular}

which was perceived to be both a cause and a result of the discharge. Multiple causal models were utilised; the most common causes were overwork and tension, two factors which were often related to each other. Biomedical causal models, including infections and contraception, were also commonly utilised. Many women sought healthcare, both biomedical and traditional, but treatments were often discontinued or changed due to lack of symptomatic relief, side-effects or costs.

\section{Interpretation of findings}

The complaint of AVD is very common, with more than 1 in 10 women experiencing it within the previous 3 months; STIs and CMDs affect about 1 in 20 women. Social disadvantage, particularly spousal sexual violence and economic difficulties, are important determinants of women's gynaecological and mental health. Current biomedical assumptions about the causes of gynaecological symptoms (for example, that the symptom of AVD is most commonly caused by STIs) are not valid in community settings. Poor mental health is an important correlate of such symptoms. Women who are suffering from CMDs and somatic complaints are more likely to develop new episodes of AVD; on the other hand, women with AVD are more likely to develop new episodes of CMDs. Thus, the relationship between AVD and poor mental health is bi-directional. The quantitative findings of this study match what women themselves tell us about their health: women closely link worries and tensions in their daily lives with their reproductive and sexual health. Several replications of this research are currently in progress, including studies in Palestine and Pakistan. One replication in Goa, with commercial sex workers, has also shown a strong association between mental ill-health and AVD (Shahmanesh et al, 2009).

Several mechanisms may explain these findings. Common mental disorders and poor mental health lead to gynaecological symptoms. We know that the most common illness experiences associated with CMDs are physical complaints. The process of somatisation, that is, the presentation of mental distress in physical forms, involves a number of mechanisms, including the misinterpretation of normal bodily experiences due to the heightened awareness associated with mental distress and heightened autonomic nervous system activity, which may increase the production of vaginal secretions. Gynaecological symptoms may be a cultural idiom to explain other physical complaints. Abnormal vaginal discharge is a genital secretion which is viewed with significance in terms of its perceived vitality in Asian cultures; in stressful life situations, women may perceive a normal physiological discharge as being abnormal, as a way to seek meaning for their unpleasant emotional and physical experiences (such as misery and tiredness, core features of CMDs) (Patel \& Oomman, 1999).

The corollary syndrome of complaints of seminal discharge has been well documented among men in South Asia (the 'dhat' syndrome). Gynaecological symptoms can also lead to CMDs; this is more straightforward to understand. Thus, if a woman is experiencing AVD, this can be a source of personal distress and worry about its cause (e.g. whether it might be an infection). Such illness experiences and worries act as stressors, which make the woman more likely to develop a 
CMD. Finally, it is plausible that both types of health problem, that is, both CMDs and AVD, are the result of severe social adversities in women's daily lives.

\section{Implications of the Stree Arogya Shodh}

The major implication of this study for health policy is that mental healthcare should be integrated in all health programmes targeted at women, in particular those for reproductive and sexual health. All health workers in reproductive and women's health programmes must be skilled in, and healthcare facilities adequately resourced for, the diagnosis and management of CMDs and somatoform disorders. There is a substantial evidence base that simple and affordable treatments (including antidepressant medicines and psychological treatments delivered by non-mental health professionals) are effective for the treatment of these disorders in low- and middle-income countries (Patel et al, 2007).

Further, there is a need to develop practical and affordable clinical algorithms for the management of AVD and STIs, and to improve the detection of CMDs; this would require the availability of cheap, bedside, diagnostic tests for STIs and bacterial vaginosis. At the very least, all primary health centres should have basic laboratory facilities and trained technicians for the diagnosis of bacterial vaginosis and should establish referral networks with appropriately equipped laboratories for diagnosis of STIs.

Programmes for mental health and reproductive health should enhance their focus on the needs of women living in socially disadvantaged circumstances, for example migrant women and those who live with violent partners. Assessment of violence should be made mandatory, particularly for married women; skills for delivering specific interventions to reduce sexual violence must be included in the training of health workers.

In conclusion, the complaint of AVD and STIs are among the most common health problems affecting women and are a priority in India's reproductive health programme. Although there is consensus that the syndromic approach is not suited to the management of either health problem, there is, as yet, no evidence-based alternative approach. A critically important research priority is to evaluate the benefits of integrating mental healthcare within reproductive health programmes, targeting the syndrome of AVD. Ultimately, this will provide the most compelling evidence with regard to the importance of integrating mental healthcare within women's reproductive and sexual health programmes.

\section{Acknowledgements}

The Stree Arogya Shodh was entirely supported by a grant from the Wellcome Trust. VP is currently a Wellcome Trust Senior Clinical Research Fellow in Tropical Medicine. A large number of collaborators participated in this study; a list of all scientific authors can be found in the publications cited in this commentary. I also acknowledge that this commentary is based on material I prepared for a full report of the findings of the study published by Sangath (see http://www.sangath. com/sangath/files/otherpdfs/sas_web.pdf)

\section{References}

Patel, V. \& Oomman, N. M. (1999) Mental health matters too: gynecological morbidity and depression in South Asia. Reproductive Health Matters, 7, 30-38

Patel, V., Kirkwood, B. R., Pednekar, S., et al (2006a) Gender disadvantage and reproductive health risk factors for common mental disorders in women: a community survey in India. Archives of General Psychiatry, 63, 404-413.

Patel, V., Kirkwood, B., Pednekar, S., et al (2006b) Why women suffer common mental disorders: a population based longitudinal study. British Journal of Psychiatry, 189, 547-555.

Patel, V., Weiss, H. A., Kirkwood, B. R., et al (2006c) Common genital complaints in women: the contribution of psychosocial and infectious factors in a population-based cohort study in Goa, India. International Journal of Epidemiology, 35, 1478-1485.

Patel, V., Araya, R., Chatterjee, S., et al (2007) Treatment and prevention of mental disorders in low-income and middle-income countries. Lancet, 370, 991-1005.

Patel, V., Andrew, G. \& Pelto, P. J. (2008) The psychological and social contexts of complaints of abnormal vaginal discharge: a study of illness narratives in India. Journal of Psychosomatic Research, 64, 255-262.

Shahmanesh, M., Wayal, S., Cowan, F., et al (2009) Suicidal behaviour among female sex workers in Goa, India: the silent epidemic. American Journal of Public Health, 99, 1239-1246.

\section{Psychiatry and mental health in Portugal}

\section{João Marques-Teixeira MD $\mathrm{PhD}^{1}$ and Elisabete Fradique $\mathrm{MD}^{2}$}

1Psychiatrist and Psychotherapist, email marquesteixeira@netcabo.pt 2Psychiatrist, email elisabete.fradique@gmail.com

Dort ortugal is in the south-west of Europe; its territory includes the Azores and Madeira islands, giving it an area of $91900 \mathrm{~km}^{2}$. The total resident population of Portugal was 10579000 in 2006 . The population density was 115 per $\mathrm{km}^{2}$. The birth rate has been declining, from 20.0 per 1000 population in 1970 to 10.4 in 2004 . Life expectancy at birth in 2006 was 75 for males and 82 years for females. Healthy life expectancy at birth in 2003 was 67 and 72 years, respectively. The infant mortality rate decreased from 10.8 per 1000 in 1991 to 3.5 per 1000 in 2005. The median age of the population has been steadily rising. 